JOURNAL OF

FUNCTION SPACES AND APPLICATIONS

Volume 7, Number 1 (2009), 25-32
(C) 2009, Scientific Horizon http://www.jfsa.net

\title{
Radial variation in some function spaces
}

\author{
David Walsh
}

(Communicated by Sten Kaijser)

2000 Mathematics Subject Classification. 30H05, 31A05, 46E15.

Keywords and phrases. Radial variation, Besov space, Lipschitz space.

Abstract. In a previous paper [8] we considered properties of the radial variation of analytic functions in a class of Besov spaces $A_{p q}^{s}, s>0$. Here we wish to extend these results to certain related spaces. These are the Lipschitz classes $\Lambda_{s}$ and the mean Lipschitz classes $\Lambda_{p, s}$ where $p \geq 1,0<s<1$. We also consider $A_{p q}^{0}$, where $s=0$, although the results obtained for these are not as good as when $s>0$.

\section{Introduction}

If $f$ is analytic in the disc, the radial variation function of $f$ is the function defined on the disc by

$$
F(r, t)=\int_{0}^{r}\left|f^{\prime}\left(u e^{i t}\right)\right| d u, \quad r<1, \quad 0 \leq t \leq 2 \pi .
$$

Since $f\left(r e^{i t}\right)-f(0)=\int_{0}^{r} f^{\prime}\left(u e^{i t}\right) d u$, it is clear that

$$
\left|f\left(r e^{i t}\right)\right| \leq|f(0)|+F(r, t), \quad r<1, \quad 0 \leq t \leq 2 \pi
$$


and $F(r, t)$ is a majorant for $f$. The function $F(r, t)$ represents the length of the image of the radius vector $\left[0, r e^{i t}\right]$ under the mapping $f$. It is clear from the definition, that the boundary function $F(t)=\lim _{r \rightarrow 1} F(r, t)$ exists, finite or infinite, for all $t \in[0,2 \pi]$. It is known as the radial or total variation. An immediate property of $F$ is that if $F(t)<\infty$, then $\lim _{r \rightarrow 1} f\left(r e^{i t}\right)$ exists.

We saw in [8] that the property that $f \in A_{p q}^{s}, 0<s<1,1 \leq p, q<\infty$, translated into meaningful results for $F$, in particular that $F(r, t)$ satisfies an analogous condition on the disc. In Section 1 we are led naturally to consider the case $s=0$ when we ask for a condition under which $F(t)$ is an integrable function on the circle. It follows immediately that $F \in L^{1}$ if and only if $f \in A_{11}^{0}$. We then show that $F(r, t)$ satisfies a corresponding condition to that by $f$ in the disc. This result extends to the general case $f \in A_{p q}^{0}$. In Section 3 we suppose that $f$ belongs to a Lipschitz space or a mean Lipschitz space and show that both $F(r, t)$ and $F(t)$ exhibit the expected behaviour.

1.1 Preliminaries. Let $D$ denote the unit disc, $T$ the unit circle in the complex plane and $L^{p}=L^{p}(T)$ the usual Lebesgue space when $0<p<\infty$. For $p \geq 1$ we denote the norm of a function $f \in L^{p}$ by $\|f\|_{p}$. For convenience we shall let $m$ denote normalised Lebesgue measure on the circle $T$.

Let $\Delta_{t} f\left(e^{i x}\right)=f\left(e^{i(x+t)}\right)-f\left(e^{i x}\right)$ and $\Delta_{t}^{m}=\Delta_{t}\left(\Delta_{t}^{m-1}\right)$. For $0<s \leq 1$, the Lipschitz class $\Lambda_{s}$ is the space of $2 \pi$-periodic functions on $[-\pi, \pi]$ for which $\left|\Delta_{t} f\left(e^{i x}\right)\right|=O\left(|t|^{s}\right)$ uniformly in $x$. A generalization is the mean Lipschitz class $\Lambda(p, s)$ consisting of all functions $f$ for which $\left\|\Delta_{t} f\right\|_{p}=$ $O\left(|t|^{s}\right)$ for $t>0 ; \Lambda(p, s)$ reduces to $\Lambda_{s}$ when $p=\infty$. Suppose now that $f$ is analytic in $D$. If $0 \leq r<1$, let

$$
M_{p}(f, r)=\left(\int_{-\pi}^{\pi}\left|f\left(r e^{i t}\right)\right|^{p} d m\right)^{1 / p}, \quad(0<p<\infty)
$$

denote the integral mean of $f$ of order $p$. It is well known that $M_{p}(f, r)$ is an increasing function of $r$ on $[0,1)$ and that the class of functions $f$ for which $\sup _{r<1} M_{p}(f, r)<\infty$, is the familiar Hardy space $H^{p}$ [2]. For $1 \leq p, q<\infty, s>0$, and an arbitrary integer $m>s$, we define the Besov space $B_{p q}^{s}$ by

$$
B_{p q}^{s}=\left\{f \in L^{p}: \int_{-\pi}^{\pi} \frac{\left\|\Delta_{t}^{m} f\right\|_{p}^{q}}{|t|^{1+s q}} d m(t)<\infty\right\} .
$$

It is well known that the definition is independent of $m$. For a discussion of these spaces see [1], [3], [4], [6], [7]. When $s$ passes through a positive 
integer value, the working definition of the Besov space $B_{p q}^{s}$ may require a change as indicated above.

The previous definition is no longer valid when $s \leq 0$; for these cases another description is required. For $n \geq 1$ we let $W_{n}$ be the polynomial on $T$ whose Fourier coefficients satisfy $\hat{W}_{n}\left(2^{n}\right)=1, \hat{W}_{n}(j)=0$ for $j \notin$ $\left(2^{n-1}, 2^{n+1}\right)$ and $\hat{W}_{n}$ is a linear function on $\left[2^{n-1}, 2^{n}\right]$ and on $\left[2^{n}, 2^{n+1}\right]$. If $n<0$ we put $W_{n}=\bar{W}_{-n}$. We put $W_{0}=\bar{z}+1+z$. For $s \leq 0,1 \leq p, q<\infty$, $B_{p q}^{s}$ consists of all distributions $f$ on $T$ for which

$$
\sum_{n=-\infty}^{\infty} 2^{|n| s}\left\|f * W_{n}\right\|_{p}^{q}<\infty .
$$

It is known that this description is equivalent to the previous one for $s>0$, but for $s=0$ in particular, only the second definition is valid. See [4] Appendix 2, [1]. In fact when $q>p$ there exist $f \in B_{p q}^{0}$ such that $f \notin L^{p}$.

Let $A_{p q}^{s}$ denote the subspace of $B_{p q}^{s}$ consisting of analytic functions. The space $A_{p q}^{s}$ for $s>0$, may be characterized as follows: for an arbitrary integer $m>s$ the analytic function $f \in A_{p q}^{s}$ if and only if

$$
\|f\|_{A}=|f(0)|+\left\{\int_{0}^{1}\left(1-r^{2}\right)^{q(m-s)-1} M_{p}\left(f^{(m)}, r\right)^{q} r d r\right\}^{1 / q}<\infty .
$$

Once again the definition is independent of $m$ for $m>s$. For $s=0$ this definition is easily modified. This is because of the property that $f \in A_{p q}^{0}$ if and only if If $\in A_{p q}^{1}$ where $I$ is the integration operator. Therefore $f \in A_{p q}^{0}$ if and only if with $m=2$,

$$
\|f\|_{A}=|f(0)|+\left\{\int_{0}^{1}\left(1-r^{2}\right)^{q-1} M_{p}\left(f^{\prime}, r\right)^{q} r d r\right\}^{1 / q}<\infty,
$$

and with $m=3$, if and only if

$$
\|f\|_{A}=|f(0)|+\left\{\int_{0}^{1}\left(1-r^{2}\right)^{2 q-1} M_{p}\left(f^{(2)}, r\right)^{q} r d r\right\}^{1 / q}<\infty .
$$

We shall need both of these representations. In particular with $p=q=1$ we have $f \in A_{11}^{0}$ if and only if

$$
\|f\|_{A}=|f(0)|+\int_{0}^{1} \int_{0}^{2 \pi}\left|f^{\prime}\left(r e^{i t}\right)\right| d m r d r<\infty .
$$




\section{Integrability of $F$}

The function $F(t)=F(1, t)$ is given from (1) by

$$
F(t)=\int_{0}^{1}\left|f^{\prime}\left(u e^{i t}\right)\right| d u, \quad 0 \leq t \leq 2 \pi .
$$

We now ask what is a sufficient condition that $F \in L^{1}$ ? Since $F \in L^{1}$ if and only if $\int_{0}^{2 \pi} \int_{0}^{1}\left|f^{\prime}\left(r e^{i t}\right)\right| r d r d m<\infty$, the answer is immediate from the definition:

Proposition 1. $F \in L^{1}(T)$ if and only if $f \in A_{11}^{0}$. Moreover

$$
\|F\|_{1}+|f(0)|=\|f\|_{A} .
$$

It may be observed here that if $f \in A_{11}^{0}$ then its boundary function $f\left(e^{i t}\right)$ exists a.e.; in fact $f \in H^{1}$. This follows by integrating the obvious inequality $\left|f\left(r e^{i t}\right)\right| \leq|f(0)|+\int_{0}^{r}\left|f^{\prime}\left(u e^{i t}\right)\right| d u$.

We can equally express the relationship in terms of the $A$-norm of $F(r, t)$. For this purpose we introduce the gradient of $F: \nabla F(r, t)=\left(\frac{\partial F}{\partial r}, 1 / r \frac{\partial F}{\partial t}\right)=$ $\left(\left|f^{\prime}\left(r e^{i t}\right)\right|, 1 / r \frac{\partial F}{\partial t}\right)$. The relationship referred to is

$$
f \in A_{11}^{0} \text { if and only if } \int_{0}^{1} \int_{0}^{2 \pi}|\nabla F(r, t)| d m r d r<\infty .
$$

If the integral is finite then it follows very simply that $f \in A_{11}^{0}$ and that $\|f\|_{A} \leq|f(0)|+\|F\|_{A}$. The proof in the other direction has already been done in essence in [8] where we considered only $s>0$. In fact we can state a more general result which follows from Theorem 1 there, and which works without any changes for our situation.

Theorem 1. Suppose that $1 \leq p, q<\infty$. There is a constant $C=$ $C(p, q)$ such that if $f \in A_{p q}^{0}$ then

$$
\int_{0}^{1}\left(1-r^{2}\right)^{q-1}\left(\int_{-\pi}^{\pi}|\nabla F(r, t)|^{p} d m\right)^{q / p} r d r \leq C\|f\|_{A}^{q} .
$$

Proof. The proof in [8] goes through word for word with $s=0$. In the case $p=q=1$ it is simpler since the use of Hölder's inequality is not needed. We do make use of the alternative definitions of $A_{p q}^{0}$ mentioned above. 
If the double integral for $F(r, t)$ is finite then as noted already it is clear that $f \in A_{p q}^{0}$. The question when $F \in L^{p}, p>1$, does not have so neat an answer. A reasonable sufficient condition is given by

Theorem 2. Suppose that $1 \leq p, q<\infty$. If $f \in A_{p 1}^{0}$ then

$$
\|F\|_{p} \leq\|f\|_{A}
$$

Proof. By Minkowski's Inequality in continuous form

$$
\begin{aligned}
\left(\int_{0}^{2 \pi}|F(t)|^{p} d m\right)^{1 / p} & =\left(\int_{0}^{2 \pi}\left(\int_{0}^{1}\left|f^{\prime}\left(r e^{i t}\right)\right| d r\right)^{p} d m\right)^{1 / p} \\
& \leq \int_{0}^{1}\left(\int_{0}^{2 \pi}\left|f^{\prime}\left(r e^{i t}\right)\right|^{p} d m\right)^{1 / p} d r \\
& <\infty
\end{aligned}
$$

and $\|F\|_{p} \leq\|f\|_{A}$.

Remark. The condition $f \in A_{p 1}^{0}$ implies that $f \in H^{p}$ for all $p \geq 1$. To see this we note that for $r<1$

$$
\left|f\left(r e^{i t}\right)\right| \leq|f(0)|+\int_{0}^{r}\left|f^{\prime}\left(u e^{i t}\right)\right| d u .
$$

On using Minkowski's Inequality again we obtain

$$
\begin{aligned}
M_{p}(f, r) & \leq|f(0)|+\int_{0}^{r} M_{p}\left(f^{\prime}, u\right) d u \\
& \leq\|f\|_{A}
\end{aligned}
$$

and the result is immediate.

In [8] it was shown that if $f \in A_{p q}^{s}, 0<s<1$, then the boundary function $F \in B_{p q}^{s}$. We do not know whether this is true for the case $s=0$ since the proof given there is no longer valid.

\section{The Lipschitz spaces}

The Lipschitz space $\Lambda_{s}, 0<s<1$, may be regarded as the Besov space $B_{\infty \infty}^{s}$. It is well known that for an analytic function $f$ on the disc, $f \in \Lambda_{s}$ if and only if there exists $M$ such that

$$
\left|f^{\prime}(z)\right| \leq \frac{M}{(1-r)^{1-s}}
$$


This property has its counterpart for the function $F(r, t)$.

Theorem 3. The function $f \in \Lambda_{s}, 0<s<1$, if and only if $\nabla F(r, t)=O\left((1-r)^{s-1}\right)$.

Proof. Suppose $f \in \Lambda_{s}$ and let $M$ be the number noted above. First we show that $F(t)$ is bounded.

$$
\begin{aligned}
F(r, t)=\int_{0}^{r}\left|f^{\prime}\left(u e^{i t}\right)\right| d u & \leq M \int_{0}^{r} \frac{1}{(1-u)^{1-s}} d u \\
& =M\left(1-(1-r)^{s}\right) / s \leq M / s
\end{aligned}
$$

for all $r<1$ and so $F(t)$ is bounded.

Since the first component of $\nabla F(r, t)$ is $\left|f^{\prime}\left(r e^{i t}\right)\right|$ we need only consider the second. Now by Lemma 3 of $[8], \frac{\partial F}{\partial t}(r, t)=\int_{0}^{r} \frac{\partial\left|f^{\prime}\right|}{\partial t}\left(u e^{i t}\right) d u$ and

$$
\begin{aligned}
\left|1 / r \frac{\partial F}{\partial t}(r, t)\right| & =\left|1 / r \int_{0}^{r} \frac{\partial\left|f^{\prime}\right|}{\partial t}\left(u e^{i t}\right) d u\right| \\
& \leq 1 / r \int_{0}^{r} u\left|f^{\prime \prime}\left(u e^{i t}\right)\right| d u \\
& \leq M \int_{0}^{r} \frac{1}{(1-u)^{2-s}} d u \leq M^{\prime} \frac{1}{(1-r)^{1-s}} .
\end{aligned}
$$

In the second inequality above we used Theorem 5.5 of [2]. The result follows.

There is a corresponding result for $F(t)$.

Theorem 4. If $f \in \Lambda_{s}, 0<s<1$, then $F(t) \in \Lambda_{s}$.

Proof. We have shown that $F$ is bounded. We write

$$
F(x)-F(t)=F(x)-F(r, x)+F(r, x)-F(r, t)+F(r, t)-F(t) .
$$

But

$$
\begin{aligned}
F(x)-F(r, x)=\int_{r}^{1}\left|f^{\prime}\left(u e^{i x}\right)\right| d u & \leq M \int_{r}^{1} \frac{1}{(1-r)^{1-s}} d u \\
& \leq M(1-r)^{s} / s
\end{aligned}
$$

and the same holds for $F(r, t)-F(t)$. Moreover $F(r, x)-F(r, t)=$ $\int_{t}^{x} \frac{\partial F}{\partial v}(r, v) d v$. Consequently 


$$
\begin{aligned}
|F(r, x)-F(r, t)| \leq\left|\int_{t}^{x}\right| \frac{\partial F}{\partial v}(r, v)|d v| & \leq M^{\prime}\left|\int_{t}^{x} \frac{1}{(1-r)^{1-s}} d v\right| \\
& =M^{\prime} \frac{1}{(1-r)^{1-s}}|t-x|,
\end{aligned}
$$

on using the previous theorem. If we now choose $1-r=|x-t|$ we get $|F(r, x)-F(r, t)| \leq M^{\prime \prime}|t-x|^{s}$ and $F(t) \in \Lambda_{s}$.

The mean Lipschitz classes $\Lambda_{p, s}(T), 1 \leq p, 0<s<1$, are indentical with the Besov spaces $B_{p \infty}^{s}$. They satisfy the condition: A function $g \in L^{p}(T)$ belongs to $\Lambda_{p, s}$ if

$$
\|g\|_{p, s}=\left(\int_{0}^{2 \pi}|g(x+t)-g(x)|^{p} d x\right)^{1 / p}=O\left(|t|^{s}\right)
$$

for small $t$. It is known (Theorem 5.4 of [2]) that an analytic function $f$ is in $\Lambda_{p, s}$ if and only if $M_{p}\left(f^{\prime}, r\right)=O\left(\frac{1}{(1-r)^{1-s}}\right) \quad 0<r<1$. With the aid of this, similar results to those of the last two theorems can be shown to hold and the proofs are straightforward.

Theorem 5. If $f \in \Lambda_{p, s}, 1 \leq p, 0<s<1$, then there exists $C=C(p, s)$ such that

(a) $\left(\int_{-\pi}^{\pi}|\nabla F(r, t)|^{p} d m\right)^{1 / p} \leq C\|f\|_{p, s}(1-r)^{s-1}$;

(b) $F(t) \in \Lambda_{p, s}$ and $\|F\|_{p, s} \leq C\|f\|_{p, s}$.

Whether a particular type of continuity for $f$ implies the same holds for $F$ is uncertain. The boundary function $f\left(e^{i t}\right)$ is absolutely continuous if and only if $f^{\prime} \in H^{1}$. We dont know that this implies that $F(t)$ is absolutely continuous but it does imply that $F$ is continuous.

Proposition 2. If $f\left(e^{i t}\right)$ is absolutely continuous then $F(t)$ is continuous.

Proof. We have $F(t+x)-F(t)=\int_{0}^{1}\left(\left|f^{\prime}\left(r e^{i(t+x)}\right)\right|-\left|f^{\prime}\left(r e^{i t}\right)\right|\right) d r$ and therefore

$$
\begin{aligned}
|F(t+x)-F(t)| & \leq \int_{0}^{1}\left|f^{\prime}\left(r e^{i(t+x)}\right)-f^{\prime}\left(r e^{i t}\right)\right| d r \\
& =\int_{0}^{1}\left|f_{x}^{\prime}\left(r e^{i t}\right)-f^{\prime}\left(r e^{i t}\right)\right| d r
\end{aligned}
$$


where $g_{x}(t)=g(t+x)$ is a translate of $g$. The Fejer-Riesz inequality allows us to conclude

$$
\begin{aligned}
\mid F(t+x) & -F(t)|+| F(t+x+\pi)-F(t+\pi) \mid \\
& \leq \int_{-1}^{1}\left|f_{x}^{\prime}\left(r e^{i t}\right)-f^{\prime}\left(r e^{i t}\right)\right| d r \leq \frac{1}{2} \int_{0}^{2 \pi}\left|f_{x}^{\prime}\left(r e^{i t}\right)-f^{\prime}\left(r e^{i t}\right)\right| d x \rightarrow 0
\end{aligned}
$$

as $x \rightarrow 0$ uniformly in $t$, because the translation map $x \rightarrow g_{x}$ is uniformly continuous from $T$ to $L^{1}$. The proof is complete.

In [8] it was seen that if we assume slightly more, namely if $f \in A_{11}^{1}$, then $F \in B_{11}^{1}$ which implies that $F$ is absolutely continuous. However mere continuity of $f$ on the circle does not even imply that $F$ is bounded. In fact Walter Rudin [5] has shown that there exists an analytic function $f$ continuous in the closed disc, such that $F(t)=\infty$ almost everywhere.

\section{References}

[1] D. Adams, and L. Hedberg, Function Spaces and Potential Theory, Springer, 1999.

[2] P.L. Duren, Theory of $H^{p}$ Spaces, Academic Press, New York, 1970.

[3] S.M. Nikolskii, Approximation of Functions of Several Variables and Embedding Theorems, Springer, 1975.

[4] V.V. Peller, Hankel Operators and Their Applications, Springer, 2003.

[5] W. Rudin, The radial variation of analytic functions, Duke Math. J., 22 (1955) 235-242.

[6] E.M. Stein, Singular Integrals and Differentiablity Properties of Functions, Princeton Univ. Press, 1970.

[7] H. Triebel, Spaces of Besov-Hardy-Sobolev Type, Teubner, Leipzig 1978.

[8] D. Walsh, Radial variation of functions in Besov spaces, Publ. Mat., 50 (2006) 371-399.

Department of Mathematics

NUI Maynooth

Ireland

(E-mail : David.Walsh@maths.nuim.ie)

(Received : January 2008) 


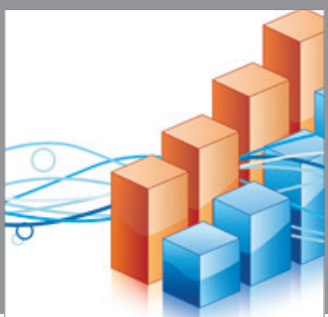

Advances in

Operations Research

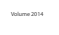

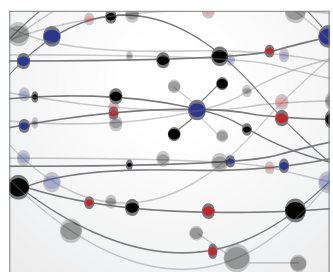

\section{The Scientific} World Journal
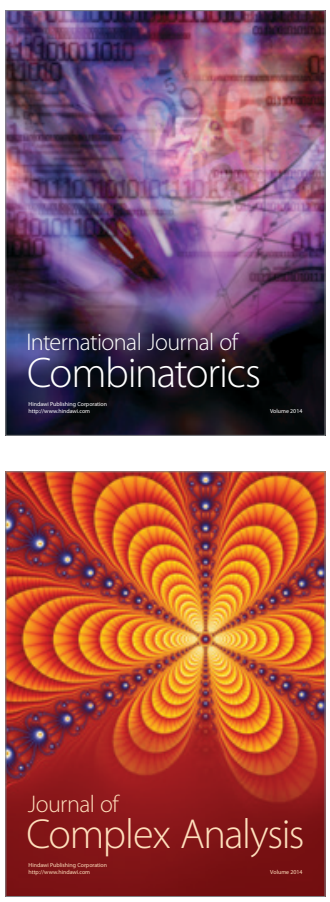

International Journal of

Mathematics and

Mathematical

Sciences
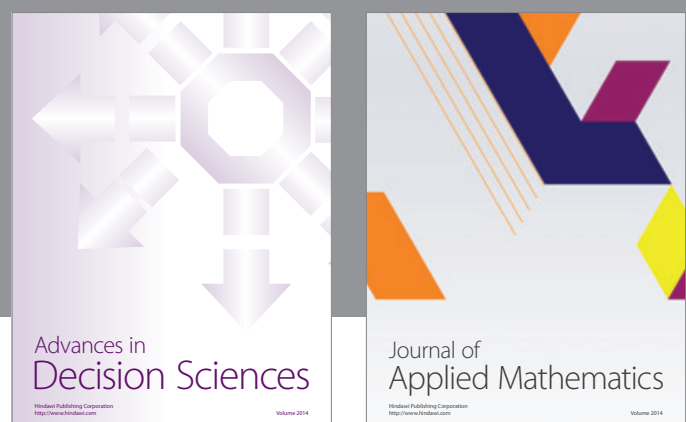

Journal of

Applied Mathematics
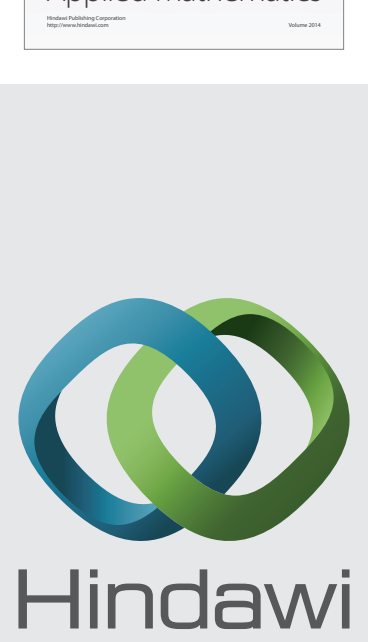

Submit your manuscripts at http://www.hindawi.com
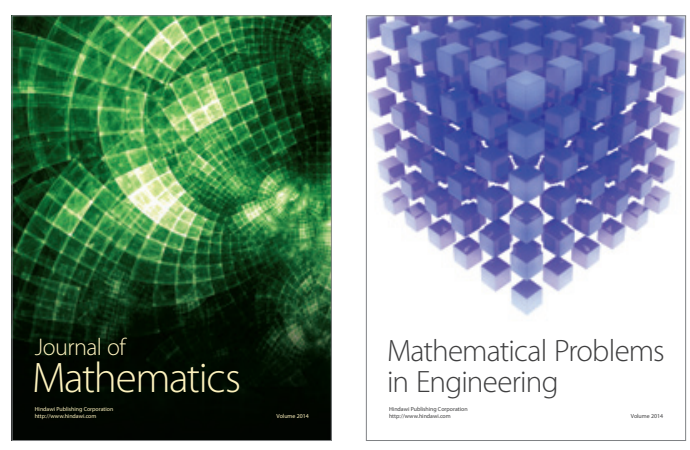

Mathematical Problems in Engineering
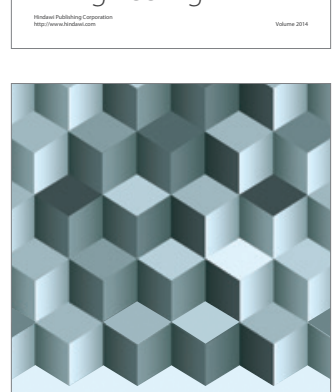

Journal of

Function Spaces
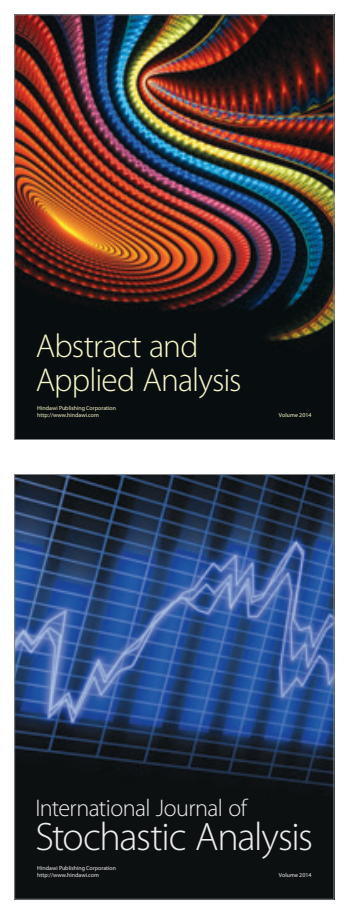

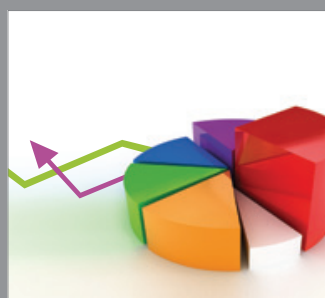

ournal of

Probability and Statistics

Promensencen
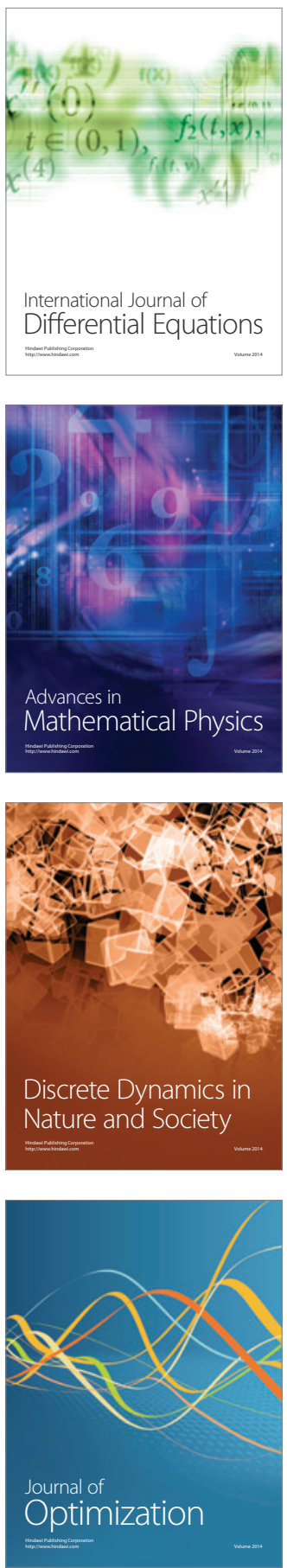